\title{
Two-Stage Channel Frequency Response Estimation in OFDM Systems
}

\author{
Oleksandr Myronchuk ${ }^{1}$, Oleksandr Shpylka ${ }^{1}$, Serhii Zhuk ${ }^{1}$ \\ ${ }^{1}$ Igor Sikorsky Kyiv Polytechnic Institute \\ 37, Prospect Peremohy, Kyiv, 03056, Ukraine
}

DOl: $10.22178 /$ pos.55-1

LCC Subject Category: TK5101-6720

Received 22.01.2019

Accepted 25.02.2020

Published online 29.02.2020

Corresponding Author:

Oleksandr Myronchuk

myronchukalex@gmail.com

(C) 2020 The Authors. This article

is licensed under a Creative

Commons Attribution 4.0 License

(c) (1)

\begin{abstract}
This paper proposes two-stage channel frequency response estimation algorithm in communication systems with OFDM technology. Algorithm is based on Kalman filter. Pilots from current and previous OFDM symbols are used for channel estimation. At the first stage data is processed in time and frequency directions. Pilots from the current OFDM symbol are filtered and at the position, where the pilots from the previous OFDM symbols should be placed, predictions are made. Predictions are based on the pilots and channel correlation characteristics. The data processing carried out on both sides relative to the array of processed data in frequency direction and on one side at processing in time direction. The results of processing are optimally combined at the second stage. The autoregressive process was used as a channel model. The analysis of the developed algorithm carried out on a model example by statistical modeling. Modeling showed that application of designed algorithm allows reducing the standard deviation of the estimation error of channel frequency response. The efficiency of designed algorithm studied using Rayleigh channel with Doppler spectrum described by Jakes model. The autocorrelation characteristics of the channel were considered as known. Modeling showed a decrease in the probability of a bit error during reception using the proposed algorithm. It is also shown that an increase in the order of the autoregressive model reduces the error in estimating the frequency response of the communication channel.
\end{abstract}

Keywords: OFDM; wireless channel; channel frequency response; channel estimation; autoregressive process; Kalman filter.

\section{INTRODUCTION}

One of the widely used technologies for transmitting information in channels with frequency selective fading is orthogonal frequency division multiplexing (OFDM). The main advantages of OFDM are high spectrum efficiency and ability to cope with severe channel conditions without complex equalization filters [1].

One of the problems that must be solved when demodulating OFDM signals is the estimation of communication channel parameters. The probability of error in reception depends on the accuracy of the channel estimate: the more accurate the estimate, the less error probability can be achieved during data transmission. This problem also exists in narrow-band communication channels, for which optimal compensation algorithms for frequency-selective distortion are proposed in [2].

Pilot signals are widely used in channel estimation. They are located in positions known to the receiver [3]. The simplest methods for estimating channel characteristics by pilots are the least square (LS) method and the method of minimum mean square error (MMSE). As established previously [4], the MMSE method has better accuracy characteristics, but it requires knowledge of the channel correlation matrix and is more laborious. A comparison of these two methods on the accuracy of estimating the channel characteristics is considered in [5].

The pilot layout in the form of a parallelogram grid is widely used in practice [6]. In case of static channels for frequency response estimation can be used the pilots from current and previous symbols. However, if channel parameters are variable in time, using the pilots from previous symbols is impossible because they contain outdated information.

In [7] an algorithm for estimating the channel frequency response based on the Kalman filter with using pilots only from the current OFDM symbol was proposed. The essence of the algo- 
rithm is that the pilots from the current symbol filtered and at the position, where the pilots from the previous OFDM symbols should be placed, predictions are made. All predictions are based on the pilots from the current symbol and the channel correlation characteristic. The data processing carried out in the frequency direction on both sides relative to the array of processed data with the subsequent optimal combination of the results.

We propose to additionally take into account the predictions based on the pilots from previous OFDM symbols. Thus, the data will be processed in both frequency and time directions at the first stage with the subsequent optimal combination of the results at the second stage (Figure 1).

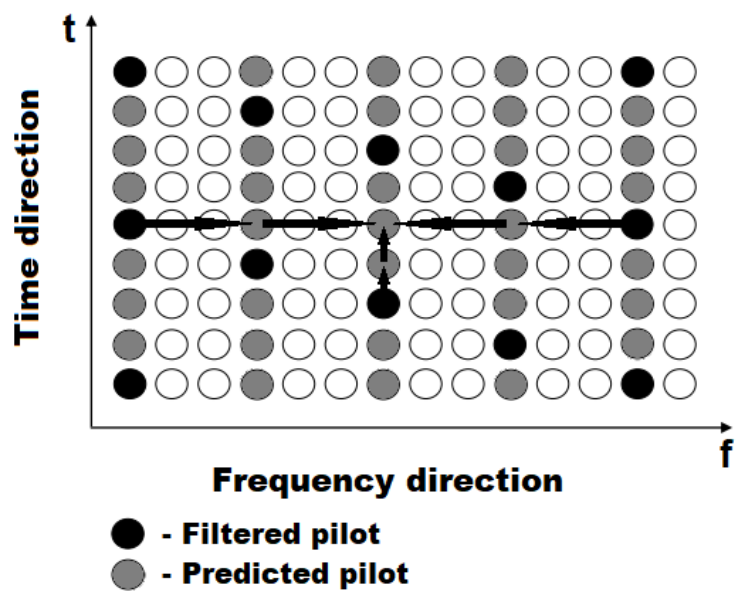

Figure 1 - The essence of designed algorithm

It is proposed to use the autoregressive (AR) process as a channel model. Studies in this area were considered in $[8,9,10]$. In particular, a method for estimating the frequency response based on the Kalman filter for a channel model in the form of a first-order AR process was propose in [8].

Problem statement. After execution the analog to digital (ADC) conversion and discrete Fourier transformation (DFT) with length $N$ the received signal takes the next form (1):

$$
\mathbf{Y}=\mathbf{X H}+\mathbf{V},
$$

where $\mathbf{Y}=\left[Y_{0}, Y_{1}, \ldots, Y_{N-1}\right]^{T}$ is a vector of received signal;

$\mathbf{X}=\operatorname{diag}\left(X_{0}, X_{1}, \ldots, X_{N-1}\right)$ is a matrix with transmitted signal on main diagonal;

$\mathbf{H}=\left[H_{0}, H_{1}, \ldots, H_{N-1}\right]^{T}$ is a vector of channel frequency response;
$\mathbf{V}=\left[V_{0}, V_{1}, \ldots, V_{N-1}\right]^{T}$ is a vector of Gaussian white noise with zero mean value and variance $\sigma_{v}^{2}$.

For further demodulation the receiver should estimate channel frequency response $\mathbf{H}$ at known transmitted signal on pilot subcarriers $\mathbf{X}$ and unknown value of noise $\mathbf{V}$.

Denote the coordinates of pilots in $n$-th OFDM symbol by plurality $\Omega_{p}^{n}=\left\{i_{0}^{n}, i_{1}^{n}, \ldots, i_{p-1}^{n}\right\}$. Thus, the equation (1) takes the following form (2):

$$
\mathbf{Y}_{n, \Omega_{p}^{n}}=\mathbf{X}_{n, \Omega_{p}^{n}} \mathbf{H}_{n, \Omega_{p}^{n}}+\mathbf{V}_{n, \Omega_{p}^{n}} .
$$

Denote the coordinates of all necessary points for channel frequency response estimation by plurality $\Omega_{e}=\left\{j_{0}, j_{1}, \ldots, j_{e-1}\right\}$ at that $\Omega_{p}^{n} \in \Omega_{e}$. Thus the task is to estimate channel frequency response in $\Omega_{e}$ positions according to available measurements $\mathbf{Y}_{n, \Omega_{p}^{n}}$.

Development of the algorithm. Any stationary random process can be represented as an infinite order AR process [8]. This property allows representing the elements of channel frequency response vector as the following autoregressive (AR) process (3):

$$
H_{j}=\sum_{k=1}^{r} \alpha_{k} H_{j-k}+\varepsilon_{j}
$$

where $\alpha_{k}$ is a coefficient of AR process;

$\varepsilon_{j}$ is a Gaussian white noise with zero mean value and variance $\sigma_{\varepsilon}^{2}$.

For filtering and extrapolation of the $n$-th OFDM symbol along the data array from left to right (forward), we introduce the system state vector for the $j$-th subcarrier of the $n$-th OFDM symbol $\mathbf{H}_{n, j}^{f}=\left[H_{n, j}^{f}, H_{n, j-1}^{f}, \ldots, H_{n, j-r+1}^{f}\right]^{T}$. Then the equation of system evolution has next form (4):

$$
\mathbf{H}_{n, j}^{f}=\mathbf{F} \mathbf{H}_{n, j-1}^{f}+\boldsymbol{\varepsilon},
$$

where $\boldsymbol{\varepsilon}=\left[\varepsilon_{n, j}, 0, \ldots, 0\right]^{T}$; 
$\mathbf{F}$ is the matrix of system evolution which has the form (5):

$$
\mathbf{F}=\left[\begin{array}{cccc}
\alpha_{1} & \alpha_{2} & \cdots & \alpha_{r} \\
1 & 0 & \cdots & 0 \\
\vdots & \ddots & \ddots & \vdots \\
0 & 0 & 1 & 0
\end{array}\right]
$$

The observation equation according to (2) takes next form (6):

$$
Y_{n, i}=\mathbf{X}_{n, i} \mathbf{H}_{n, i}^{f}+V_{n, i}
$$

where $i \in \Omega_{p}^{n} ; \mathbf{X}_{n, i}=\left[\begin{array}{llll}X_{n, i} & 0 & \cdots & 0\end{array}\right]$;

$Y_{n, i}$ is the observation corresponding to the $i$-th subcarrier of the $n$-th OFDM symbol.

According to the model (4), (6), algorithm of filtration and extrapolation describes by the next equations (7-10):

$$
\begin{gathered}
\mathbf{H}_{n, j}^{* f}=\mathbf{F} \hat{\mathbf{H}}_{n, j-1}^{f}, \\
\mathbf{P}_{n, j}^{* f}=\mathbf{F} \hat{\mathbf{P}}_{n, j-1}^{f} \mathbf{F}^{T}+\mathbf{Q}, \\
\left\{\begin{array}{c}
\hat{\mathbf{H}}_{n, j}^{f}=\mathbf{H}_{n, j}^{* f}, \text { if } j \notin \Omega_{p}^{n} \\
\hat{\mathbf{H}}_{n, j}^{f}=\mathbf{H}_{n, j}^{* f}+\mathbf{K}_{n, j}^{f}\left(Y_{n, j}-\mathbf{X}_{n, j} \mathbf{H}_{n, j}^{* f}\right), \text { if } j \in \Omega_{p}^{n}
\end{array}\right. \\
\left\{\begin{array}{c}
\hat{\mathbf{P}}_{n, j}^{f}=\mathbf{P}_{n, j}^{* f}, \text { if } j \notin \Omega_{p}^{n} \\
\hat{\mathbf{P}}_{n, j}^{f f}=\mathbf{P}_{n, j}^{* f}-\mathbf{K}_{n, j}^{f} \mathbf{X}_{n, j} \mathbf{P}_{n, j}^{* f}, \text { if } j \in \Omega_{p}^{n}
\end{array}\right.
\end{gathered}
$$

where $\mathbf{Q}=\operatorname{diag}\left(\sigma_{\varepsilon}^{2}, 0, \ldots, 0\right)$;

$\mathbf{H}_{n, j}^{* f}, \hat{\mathbf{H}}_{n, j}^{f}$ are prediction and estimation vectors of $\mathbf{H}_{n, j}^{f} ; \mathbf{P}_{n, j}^{* f}, \hat{\mathbf{P}}_{n, j}^{f}$ are correlation matrices of prediction and estimation errors;

$\mathbf{K}_{n, j}^{f}=\mathbf{P}_{n, j}^{* f} \mathbf{X}_{n, j}^{T} /\left(\mathbf{X}_{n, j} \mathbf{P}_{n, j}^{* f} \mathbf{X}_{n, j}^{T}+\sigma_{v}^{2}\right)$ is a vector of gain coefficients which is calculated only at points $j \in \Omega_{p}^{n}$.

Variance $\sigma_{\varepsilon}^{2}$ is determined as (11):

$$
\sigma_{\varepsilon}^{2}=\sigma^{2}-\mathbf{A}^{T} \mathbf{R}^{-1} \mathbf{A}
$$

where $\sigma^{2}$ is a variance of frequency response values;

$\mathbf{R}$ is a channel correlation matrix, which has the form:

$$
\begin{gathered}
\mathbf{R}=\left[\begin{array}{cccc}
\sigma^{2} & \operatorname{conj}\left(R_{1}\right) & \cdots & \operatorname{conj}\left(R_{r-1}\right) \\
R_{1} & \sigma^{2} & \cdots & \operatorname{conj}\left(R_{r-2}\right) \\
\vdots & \vdots & \ddots & \vdots \\
R_{r-1} & R_{r-2} & \cdots & \sigma^{2}
\end{array}\right] \\
\mathbf{A}=\left[\begin{array}{c}
R_{1} \\
R_{2} \\
\vdots \\
R_{r}
\end{array}\right] .
\end{gathered}
$$

For filtering and extrapolation from right to left (backward) algorithm is similar. Moreover, vectors $\mathbf{H}_{n, j}^{* b}, \hat{\mathbf{H}}_{n, j}^{b}$ and matrices $\mathbf{P}_{n, j}^{* b}, \hat{\mathbf{P}}_{n, j}^{b}$ are determined at each point. An array of processed OFDM symbol data and processing directions is presented in the Figure 2.

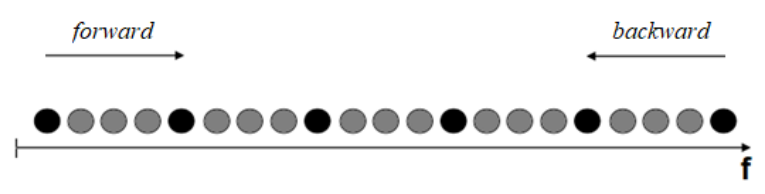

Figure 2 - The view of data array and processing directions

Following the technique given in [11] the algorithm for optimal combining the processing of the results in forward and backward directions at point $j$ is described by the next equations (1215):

$$
\begin{aligned}
& \tilde{P}_{n, j}=P_{n, j}^{* f}-\frac{P_{n, j}^{* f 2}}{\left(\hat{P}_{n, j}^{b}+P_{n, j}^{* f}\right)}, \\
& \tilde{H}_{n, j}=\hat{H}_{n, j}^{b}+\frac{\tilde{P}_{n, j}}{P_{n, j}^{* f}} \cdot\left(H_{n, j}^{* f}-\hat{H}_{n, j}^{b}\right),
\end{aligned}
$$

$$
\breve{P}_{n, j}=-\bar{P}-\frac{\bar{P}^{2}}{\left(\tilde{P}_{n, j}-\bar{P}\right)},
$$




$$
\breve{H}_{n, j}=\tilde{H}_{n, j}-\frac{\breve{P}_{n, j}}{\bar{P}} \cdot\left(\bar{H}-\tilde{H}_{n, j}\right),
$$

where $\hat{H}_{n, j}^{b}, H_{n, j}^{* f}$ are elements with index (1) of vectors $\hat{\mathbf{H}}_{n, j}^{b}$ and $\mathbf{H}_{n, j}^{* f}$;

$\hat{P}_{n, j}^{b}, P_{n, j}^{* f}$ are elements with indexes $(1,1)$ of matrices $\hat{\mathbf{P}}_{n, j}^{b}$;

$\mathbf{P}_{n, j}^{* f} ; \bar{H}, \bar{P}$ are a priori known mean value and variance of channel frequency response;

$\breve{H}_{n, j}$ is a combined estimation;

$\breve{P}_{n, j}$ is an error of combined estimation.

Similarly, the algorithm applied on all subcarriers from the set $\Omega_{e}$ for filtering and extrapolation in the time direction. The changing of the frequency response values on the $j$-th subcarrier in time can be represented as an h order AR process (16):

$$
H_{n}=\sum_{k=1}^{h} \beta_{k} H_{n-k}+\varepsilon_{n},
$$

where $\beta_{k}$ is a coefficient of AR process;

$\varepsilon_{n}$ is a Gaussian white noise with zero mean value and variance $\sigma_{\varepsilon}^{2}$.

For filtering and extrapolation values at $j$-th subcarrier in time we introduce the system state vector at the $n$-th time moment $\mathbf{H}_{n, j}^{t}=\left[H_{n, j}^{t}, H_{n-1, j}^{t}, \ldots, H_{n-h+1, j}^{t}\right]^{T}$. Then the equation of system evolution has next form (17):

$$
\mathbf{H}_{n, j}^{t}=\mathbf{F} \mathbf{H}_{n-1, j}^{t}+\boldsymbol{\varepsilon},
$$

where $\boldsymbol{\varepsilon}=\left[\varepsilon_{n, j}, 0, \ldots, 0\right]^{T}$;

$\mathbf{F}$ is the matrix of system evolution which has the next form (18):

$$
\mathbf{F}=\left[\begin{array}{cccc}
\beta_{1} & \beta_{2} & \cdots & \beta_{h} \\
1 & 0 & \cdots & 0 \\
\vdots & \ddots & \ddots & \vdots \\
0 & 0 & 1 & 0
\end{array}\right]
$$

The observation equation takes next form (19):

$$
Y_{n, i}=\mathbf{X}_{n, i} \mathbf{H}_{n, i}^{t}+V_{n, i},
$$

where $i \in \Omega_{p}^{n} ; \mathbf{X}_{n, i}=\left[\begin{array}{llll}X_{n, i} & 0 & \cdots & 0\end{array}\right]$;

$Y_{n, i}$ is the observation corresponding to the $i$-th subcarrier of the $n$-th OFDM symbol.

According to the model (17), (19), algorithm of filtration and extrapolation describes by the next equations (20-23):

$$
\begin{aligned}
& \mathbf{H}_{n, j}^{* t}=\mathbf{F} \hat{\mathbf{H}}_{n-1, j}^{t}, \\
& \mathbf{P}_{n, j}^{*_{t}}=\mathbf{F} \hat{\mathbf{P}}_{n-1, j}^{t} \mathbf{F}^{T}+\mathbf{Q}, \\
& \left\{\begin{array}{c}
\hat{\mathbf{H}}_{n, j}^{t}=\mathbf{H}_{n, j}^{*_{t}}, \text { if } j \notin \Omega_{p}^{n} \\
\hat{\mathbf{H}}_{n, j}^{t}=\mathbf{H}_{n, j}^{* t}+\mathbf{K}_{n, j}^{t}\left(Y_{n, j}-\mathbf{X}_{n, j} \mathbf{H}_{n, j}^{* t}\right), \text { if } j \in \Omega_{p}^{n}
\end{array}\right. \\
& \left\{\begin{array}{c}
\hat{\mathbf{P}}_{n, j}^{t}=\mathbf{P}_{n, j}^{* t}, \text { if } j \notin \Omega_{p}^{n} \\
\hat{\mathbf{P}}_{n, j}^{t}=\mathbf{P}_{n, j}^{* t}-\mathbf{K}_{n, j}^{t} \mathbf{X}_{n, j} \mathbf{P}_{n, j}^{* t}, \text { if } j \in \Omega_{p}^{n}
\end{array}\right.
\end{aligned}
$$

where $\mathbf{Q}=\operatorname{diag}\left(\sigma_{\varepsilon}^{2}, 0, \ldots, 0\right)$;

$\mathbf{H}_{n, j}^{* t}, \hat{\mathbf{H}}_{n, j}^{t}$ are prediction and estimation vectors of $\mathbf{H}_{n, j}^{t}$;

$\mathbf{P}_{n, j}^{* t}, \hat{\mathbf{P}}_{n, j}^{t}$ are correlation matrices of prediction and estimation errors;

$\mathbf{K}_{n, j}^{t}=\mathbf{P}_{n, j}^{* t} \mathbf{X}_{n, j}^{T} /\left(\mathbf{X}_{n, j} \mathbf{P}_{n, j}^{* t} \mathbf{X}_{n, j}^{T}+\sigma_{v}^{2}\right)$ is a vector of gain coefficients which is calculated only at points $j \in \Omega_{p}^{n}$. The variance $\sigma_{\varepsilon}^{2}$ is determined by equation (11).

To combine the results obtained from (14)-(15) and (20)-(23), the following algorithm is used (24-27):

$$
\begin{aligned}
& \widehat{P}_{n, j}=P_{n, j}^{* t}-\frac{P_{n, j}^{* t 2}}{\left(\breve{P}_{n, j}+P_{n, j}^{* t}\right)}, \\
& \hat{H}_{n, j}=\breve{H}_{n, j}+\frac{\widehat{P}_{n, j}}{P_{n, j}^{* t}} \cdot\left(H_{n, j}^{* t}-\breve{H}_{n, j}\right),
\end{aligned}
$$




$$
\begin{aligned}
& P_{n, j}^{c}=-\bar{P}-\frac{\bar{P}^{2}}{\left(\widehat{P}_{n, j}-\bar{P}\right)}, \\
& H_{n, j}^{c}=\hat{H}_{n, j}-\frac{P_{n, j}^{c}}{\bar{P}} \cdot\left(\bar{H}-\widehat{H}_{n, j}\right) .
\end{aligned}
$$

\section{RESULTS AND DISCUSSION}

The analysis of the developed algorithm is carried out on a model example by statistical modeling. The frequency response of the channel represented by a linear Gaussian AR model [12]:

$H_{1,1}=\sqrt{\sigma^{2}} \cdot \xi_{1,1}$

$H_{m, 1}=\alpha_{1} H_{m-1,1}+\sqrt{\sigma^{2}\left(1-\operatorname{conj}\left(\alpha_{1}\right) \cdot \alpha_{1}\right)} \cdot \xi_{m, 1}, m=2 \ldots N_{m}$,

$H_{1, n}=\beta_{1} H_{1, n-1}+\sqrt{\sigma^{2}\left(1-\operatorname{conj}\left(\beta_{1}\right) \cdot \beta_{1}\right)} \cdot \xi_{1, n}, n=2 \ldots N_{n}$,

$H_{m, n}=\alpha_{1} H_{m-1, n}+\beta_{1} H_{m, n-1}-\alpha_{1} \beta_{1} H_{m-1, n-1}+\sqrt{\sigma^{2}\left(1-\operatorname{conj}\left(\alpha_{1}\right) \cdot \alpha_{1}\right)\left(1-\operatorname{conj}\left(\beta_{1}\right) \cdot \beta_{1}\right)} \cdot \xi_{m, n}$,

$m=2 \ldots N_{m}, n=2 \ldots N_{n}$

where $\xi$ is a Gaussian white noise.

Model parameters: $\alpha_{1}=0.95-0.05 i, \beta_{1}=0.85, \sigma^{2}=0.9$ are believed to be known. Variance of the noise $\sigma_{v}^{2}=0.01$. Size of investigated channel in frequency direction $N_{m}=1024$ points; in time direction $N_{n}=512$ points.

Figure 3 shows theoretical $\sigma^{\prime}$ standard deviations of estimation error calculated based on formula (10) for processing in the forward and backward directions; based on formula (14) for results of optimal combining forward + backward; based on formula (26) for results of optimal combining forward + backward + time.

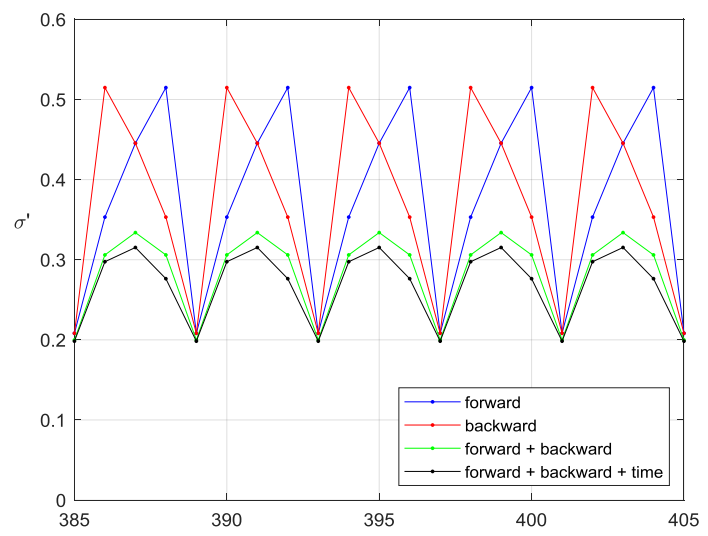

Figure 3 - Theoretical standard deviations of estimation error

Figure 4 shows experimental standard deviations of estimation error obtained by Monte Carlo method. For clarity, the results of the algorithm on an interval of 20 points from the total number of points processed are displayed.

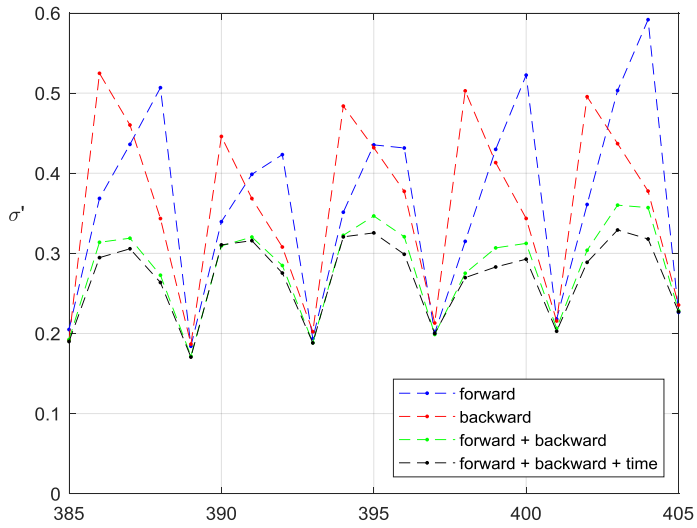

Figure 4 - Experimental standard deviations of estimation error

As can be seen from the plots, theoretical and experimental results agree, which confirms the validity of the algorithm. Moreover, the algorithm (12)-(15) allows to decrease the standard deviation of the estimation error by $50 \%$ in comparison with algorithm (7)-(10). Application of algorithm (24)-(27) allows to further reduce the standard deviation of the estimation error by 10\% compared with algorithm (12)-(15).

The efficiency of designed algorithm is studied using Rayleigh channel with Doppler spectrum that describes by Jakes model [13]. Coefficients of AR process are obtained by Yule-Walker equa- 
tions are obtained from channel autocorrelation characteristic. The autocorrelation characteristic of the channel is considered known.

Figure 5 shows dependence of the mean square error (MSE) of the channel estimate on variance of the noise for communication channel with 20 rays, $10^{\mathrm{e}-6} \mathrm{~s}$ maximum delay, $10 \mathrm{~Hz}$ Doppler shift. The autoregressive models of the first and seventh order in frequency and time directions were used.

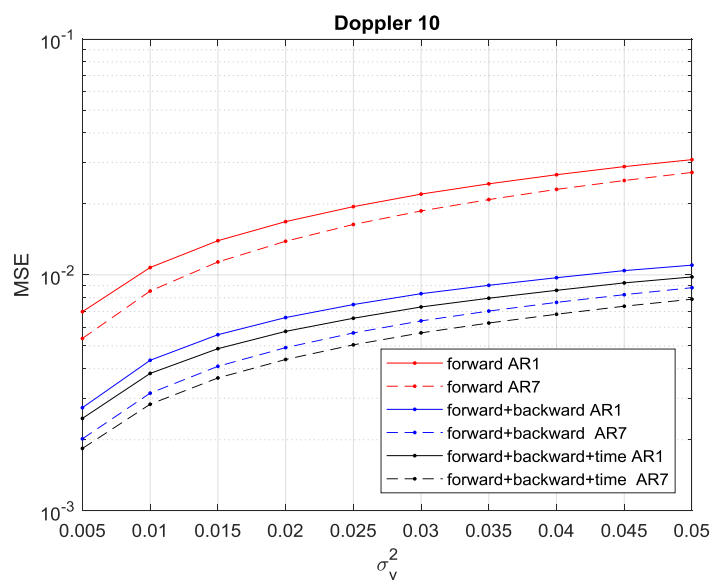

Figure 5 - Mean square error -Doppler $10 \mathrm{~Hz}$

Similar dependences for a communication channel with a Doppler shift of $50 \mathrm{~Hz}$ shown at Figure 6.

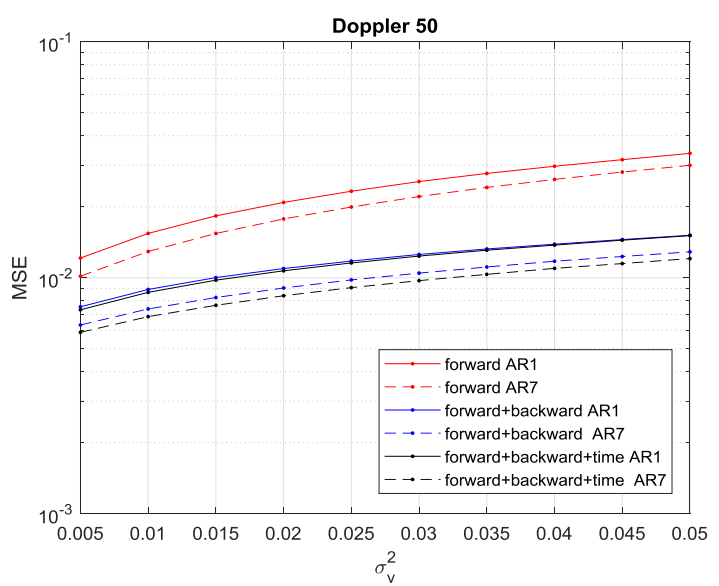

Figure 6 - Mean square error -Doppler $50 \mathrm{~Hz}$

As can be seen from the above plots, an increase in the order of the autoregressive model reduces the error in estimating the frequency response of the communication channel.

Figure 7 shows dependence of the bit error rate (BER) on signal to noise ratio (SNR) for channel with $10 \mathrm{~Hz}$ Doppler shift when using QPSK modulation on OFDM symbol subcarriers.

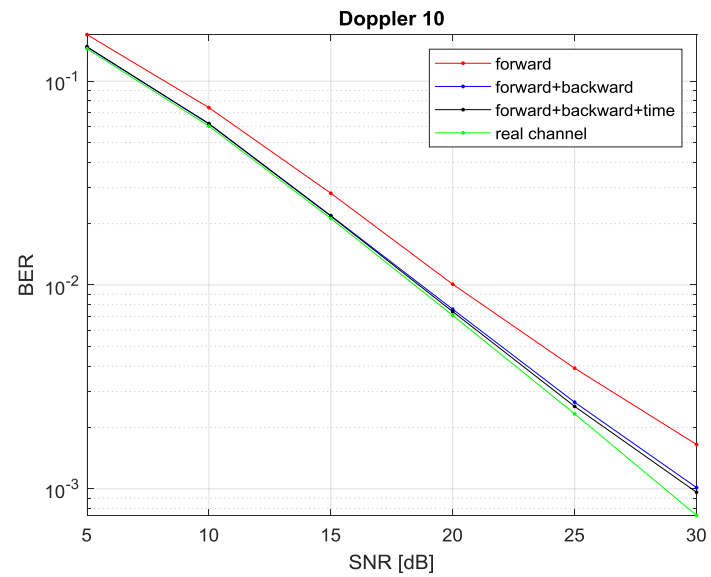

Figure 7 - Bit error rate -Doppler $10 \mathrm{~Hz}$

Similar dependences for a communication channel with a Doppler shift of $50 \mathrm{~Hz}$ shown at Figure 8 .

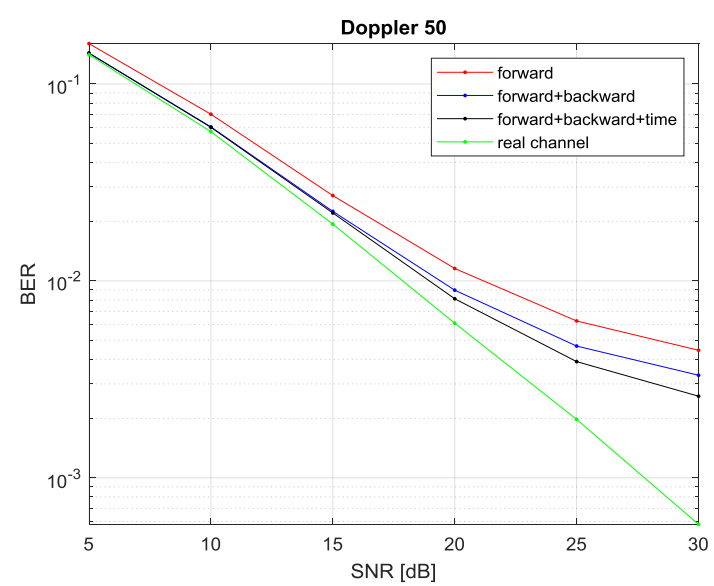

Figure 8 - Bit error rate -Doppler $50 \mathrm{~Hz}$

In Figure 7 and Figure 8 green line shows a curve of theoretically achievable BER values for a known channel frequency response. As can be seen from the plots, the application of algorithm (12)-(14) gives a gain of up to $2,5 \mathrm{~dB}$ in comparison on algorithm (7)-(10) in a channel with a Doppler shift of $50 \mathrm{~Hz}$ and up to $2 \mathrm{~dB}$ in a channel with a Doppler shift of $10 \mathrm{~Hz}$. Application of algorithm (24)-(27) gives an additional gain of up to $1 \mathrm{~dB}$ in a channel with a Doppler shift of $50 \mathrm{~Hz}$ and up to $0.5 \mathrm{~dB}$ in a channel with a Doppler shift of $10 \mathrm{~Hz}$.

\section{CONCLUSIONS}

Thus, the proposed algorithm makes the optimal combination of the three results of estimation the frequency response of the communication channel, which is represented by the autoregressive model. The estimation is based on the Kalman filtering theory using pilot signals and the known correlation characteristics of the communication 
channel. Two estimates for the point, at which the combining is made, are obtained using the pilot signals of the current symbol by filtration and extrapolation in both sides in frequency direction. The third estimate is obtained using the measurements obtained in the previous symbols, which corresponds to the coordinate of the given point. Modeling showed a decrease in the probability of a bit error during reception using the proposed algorithm. A further area of the research is the development of an algorithm for jointly estimating the frequency response of a communication channel and decision making on transmitted symbol.

\section{REFERENCES}

1. Rohling, H. (Ed.). (2011). OFDM. Signals and Communication Technology. doi: 10.1007/978-3-64217496-4

2. Shpylka, A. A., \& Zhuk, S. Y. (2010). Joint interpolation of data and parameter filtration of a multibeam communications channel. Radioelectronics and Communications Systems, 53(1), 20 24. doi: $10.3103 / \mathrm{s} 0735272710010048$

3. Chiueh, T.-D., Tsai, P.-Y., \& Lai, I.-W. (2012). Baseband Receiver Design for Wireless MIMO-OFDM Communications (2nd ed.). Singapore: John Wiley \& Sons.

4. Shen, Y., \& Martinez, E. (2006). Channel estimation in OFDM systems. Retrieved from https://pdfs.semanticscholar.org/bb01/a1cf553eeb6d9101b77d0ebe92fb44172347.pdf?_ga=2. 176146658.1983668023.1582973234-1042230176.1581878685

5. Van de Beek, J.-J., Edfors, O., Sandell, M., Wilson, S. K., \& Borjesson, P. O. (n. d.). On channel estimation in OFDM systems. 1995 IEEE 45th Vehicular Technology Conference. Countdown to the Wireless Twenty-First Century. doi: 10.1109/vetec.1995.504981

6. Fazel, K., \& Kaiser, S. (2009). Multi-carrier and spread spectrum systems: from OFDM and MC-CDMA to LTE and WiMAX (2nd ed.). Hoboken: John Wiley \& Sons.

7. Myronchuk, A. Y., Shpylka, O. O., \& Zhuk, S. Y. (2019). Channel frequency response estimation method based on pilot's filtration and extrapolation. Visnyk NTUU KPI Seriia - Radiotekhnika Radioaparatobuduvannia, 78, 36-42. doi: 10.20535/radap.2019.78.36-42

8. Ki-Young Han, Sang-Wook Lee, Jun-Seok Lim, \& Koeng-Mo Sung. (2004). Channel estimation for OFDM with fast fading channels by modified Kalman filter. IEEE Transactions on Consumer Electronics, 50(2), 443-449. doi: 10.1109/tce.2004.1309406

9. Huang, M., Chen, X., Xiao, L., Zhou, S., \& Wang, J. (2007). Kalman-filter-based channel estimation for orthogonal frequency-division multiplexing systems in timevarying channels. IET Communications, 1(4), 795-801.

10. Wei Chen, \& Ruifeng Zhang. (n.d.). Kalman-filter channel estimator for OFDM systems in time and frequency-selective fading environment. 2004 IEEE International Conference on Acoustics, Speech, and Signal Processing. doi: 10.1109/icassp.2004.1326842

11. Vishnevyy, S. V., \& Zhuk, S. Y. (2011). Two-stage mutual causal filtration and segmentation of heterogeneous images. Radioelectronics and Communications Systems, 54(1), 37-44. doi: $10.3103 / \mathrm{s} 0735272711010067$

12. Favorskaya, M., \& Jain, L. (2020). Computer Vision in Advanced Control Systems-5: Advanced Decisions in Technical and Medical Applications. Cham: Springer International Publishing.

13. Jakes, W. C. (Ed). (1994). Microwave Mobile Communications. New York: IEEE Press. 